\title{
Penetrating wound of the orbit: a 31-year follow-up
}

\author{
MILTON J. FREIWALD \\ Philadelphia, Pennsylvania, USA
}

SUMMARY The case is described of a man who at the age of 17 suffered accidental penetration of his right orbit by a rod and spring from a machine gun. Operation was successful, and follow-up 31 years later showed normal vision at 20/20 in both eyes.

Penetrating wounds of the orbit may cause considerable damage, resulting in the following complications: rupture of the eyeball with expulsion of the intraocular contents; retinal separation, immediate or delayed; avulsion of the optic nerve, with permanent blindness; ocular muscle injury of such severity as to cause permanent diplopia, lachrymal gland injury as well as extensive fractures of the or bital walls into the sinus cavities or into the cranial cavity, with leakage of the cerebrospinal fluid, a threat to life. Penetrating orbitocranial injuries are a grave threat to the patient's survival, with $12 \%$ mortality among 42 cases of orbitocranial wounds during the second world war (Webster and Guardjian, 1943). Serious infections may be introduced, with resulting meningitis, intracranial haemorrhage, and cavernous sinus thrombosis resulting in a fatal outcome.

On the other hand gross injuries may cause astonishingly little damage and often leave the eyeball intact along with other vital structures. Many bizarre injuries have been reported (Duke-Elder, 1954) wherein various foreign bodies of different sizes, shapes, and edges pass through the orbit without damaging any important structures. Uneventful recovery may follow without functional impairment.

A successful outcome of these injuries depends on the following important factors: the path of the penetrating object, the size of the object, the space the object takes in a constricted area, the time factor (from the time of injury to the time of medical and surgical care), the extent of the surgical intervention, the elimination of all possible complications, and the routes of antibiotic therapy to prevent or control secondary infection. A careful examination of the anterior and posterior ocular

Address for reprints: $\operatorname{Dr}$ M. F. Freiwald, c/o George F. Stickley Co, 210W Washington Square, Philadelphia, Pa 19106, USA segments for any sign of haemorrhage, laceration, or oedema are important to the medical management.

\section{CASE REPORT}

On 13 May 1947 a 17-year-old Caucasian welldeveloped and well-nourished enlisted man in the US Army was brought from a training area to the Tilton General Hospital at Fort Dix, New Jersey, on a litter. He had been accidentally struck by a rod and spring from a BAR (Browning automatic rifle) machine gun during a class problem of reassembling the gun (Fig. 1). At the time he was getting the gun ready for firing when the trigger mechanism was set off. The driving spring and rod became disengaged from the belt and were forcibly ejected to the rear of the expanding drive spring. The spring and rod together struck and penetrated his right orbit (Fig. 2).

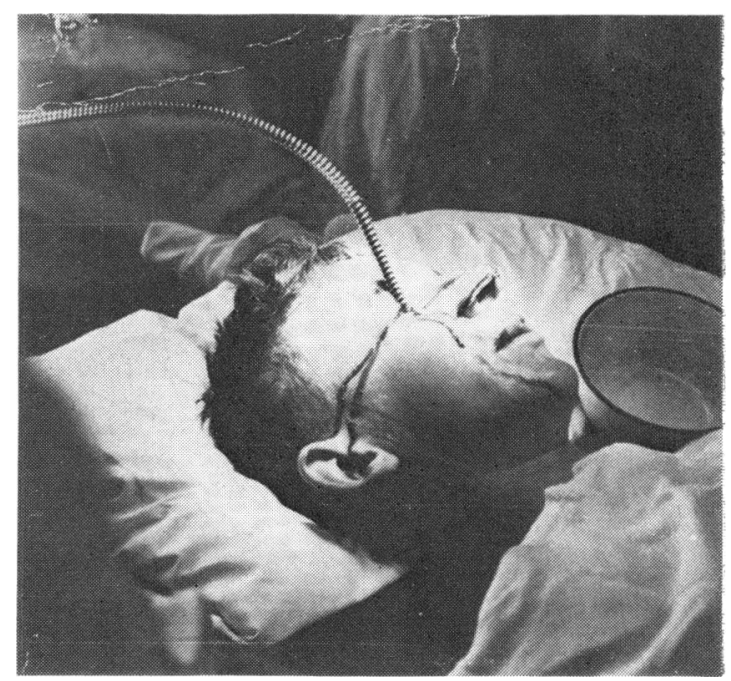

Fig. 1 Patient when admitted to hospital 


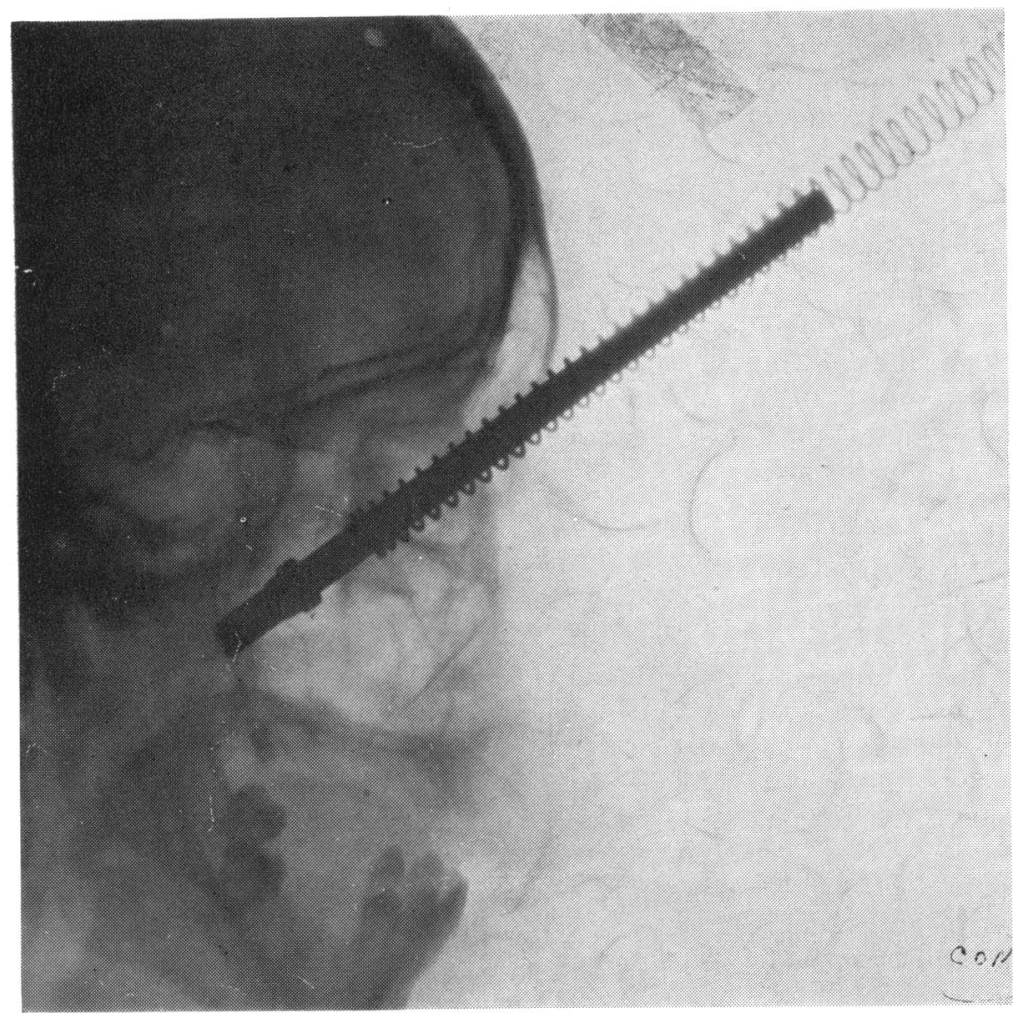

Fig. 2 Radiograph showing spring and rod in patient's orbit

The patient was taken immediately to hospital, where morphine was administered intramuscularly. He was scheduled for surgery within three-quarters of an hour from the time of the accident. On examination he was seen to be in acute distress though conscious, with a spring approximately 12 in $(30 \mathrm{~cm})$ long and a central steel rod of approximately $6 \frac{1}{2}$ in $(16 \mathrm{~cm})$ of which 2 in $(5 \mathrm{~cm})$ was projecting from the right orbit. Blood was splattered about the face and base of the spring. The right eyelid was everted to determine the extent of damage to the eyeball. Strangely enough the right eyeball was markedly compressed and wedged against the lateral orbital wall (the greater wing of the sphenoid bone).

Portable $x$-ray films showed the entrance path of the foreign body through the nasal floor of the right orbit, passing through the right antrum, then through the ethmoid sinus, and lodging in the pharyngeal wall.

Under general anaesthesia a small metallic plate was carefully passed between the spring and eyeball to prevent ethmoid bone spicules from lacerating the eyeball on removal of the spring. Gentle pressure was applied in the pharynx against the rod, and the spring was unwound counterclockwise to a complete and safe withdrawal, with minimal bleeding. The spring and rod were contaminated with an oily substance and bony spicules between the coils of the spring.

Anticipating the problem of chronic sinusitis, plus the need for adequate drainage, a Caldwell-Luc procedure was carried out. Numerous fragments of bone were removed from the right antrum, and a rubber drain was inserted to allow for drainage, so that any postoperative suppuration would eliminate the possibility of cavernous sinus thrombosis or meningitis. The patient was given 10 million units of aqueous penicillin intramuscularly. The eyegrounds showed a moderately severe degree of retinal haemorrhage but no clinical evidence of retinal separation or damage to the optic nerve or the macular area. The anterior segment appeared normal, and there was no evidence of hyphaemia. The iris was not torn, and the intraocular pressure was within normal limits, by the digital pressure method, $18 \mathrm{mmHg}$ for both eyes. Atropine sulphate $1 \%$ was instilled in the right eye to keep the pupil open. Iodides were administered orally to absorb the retinal haemorrhages.

The postoperative course was uneventful, and during the next 7 days normal healing took place 
with no evidence of infection, global, sinus, or central. There was some evidence of diplopia, but as the oedema subsided it disappeared. During the following 2 weeks there were good extraocular muscle movements in all cardinal directions of gaze. The retinal haemorrhages cleared, with resultant vision of $20 / 20$ and normal visual fields, taken on the tangent screen. On the 10th hospital day penicillin dosage was reduced to 3.6 million units intramuscularly per day for the next 10 days and then stopped. All vital signs and blood studies were found to be normal.

On the 26th hospital day the antral window closed, with no evidence of complications, and on 10 June, 27 days after operation, the patient was given leave for 20 days' bed rest at his home. On 7 July he returned from leave complaining of blurred vision in his right eye. Examination revealed a massive retinal separation, the right retina hanging like a balloon from the superior and temporal wall, extending from 8 o'clock to 1 o'clock clockwise. On close questioning it was learned he had been swimming and diving almost every day of his leave; repeated concussions may have caused the retinal separation.

The patient was put on bed-rest, and on 9 July 1947 was operated on for repair of the retinal separation, with disinsertion by the Walker method (creating electro-inflammation with small multiple needles through the sclera) around the periphery of the retinal separation, thus causing a sealed scar and preserving the rest of the retina for restoration of vision in the right eye. He made an uneventful recovery, with the excellent result of $20 / 20$ vision and ability to read small print (Fig. 3). On 14 August 1947 the patient was discharged from the hospital and honourably separated from the army.

On 19 February 1977 this patient visited my office for a check-up after 31 years. He is now 48 years old and has no complaints. He has had no problems with his health and particularly his eye or sinuses. $\mathrm{He}$ and his wife have reared 9 children.

Examination now shows normal vision, right eye $20 / 20$, left eye $20 / 20$. The eyegrounds reveal normal retina and normal retinal vascular tree, except for the area extending from 8 to 1 o'clock clockwise in the periphery, which is sealed off by scar and pigmentation, exactly as it was when he was operated on by me 31 years ago. The vitreous is clear, and the optic nerve and macula are clear and normal.

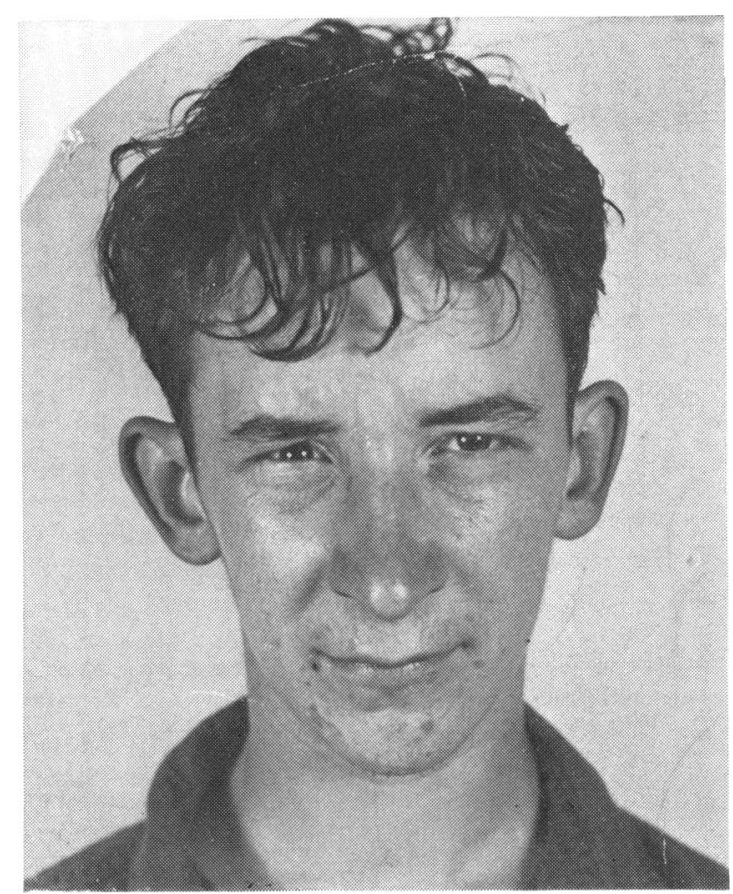

Fig. 3 Patient on discharge from hospital in 1947

The visual field is normal except for the scotoma inferiorly and nasally consistant with the repaired retina at the time of the original surgery.

\section{Discussion}

The significant aspects of this dramatic recovery from traumatic injury are, first, that the successful outcome mainly depended on the speed with which the patient was treated. Secondly, from the time he was injured to the time of surgical removal of the rod and spring was only three-quarters of an hour. Thirdly, the retinal separation was at a favourable location. The Caldwell-Luc operation for drainage and the extensive use of antibiotics contributed to his recovery.

\section{References}

Duke-Elder, S. (1954). Textbook of Ophthalmology, Vol. 6, p. 6114. Kimpton: London.

Webster, J. L., and Guardjian, E. S. (1943). Acute physiologic effects of gunshot and other penetrating wounds of the brain. Journal of Neurophysiology, 6, 225-262. 